$$
\begin{aligned}
& \text { تقييم ومقارنة أداء محرك ديزل وملوثاتها يعمل بعدة خلائط لوقود }
\end{aligned}
$$

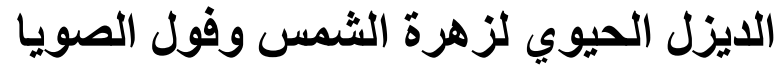

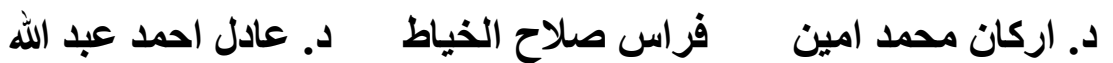

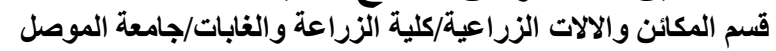

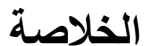

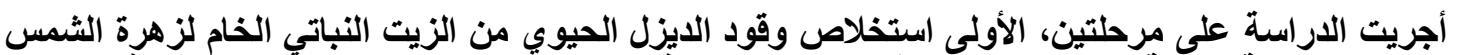

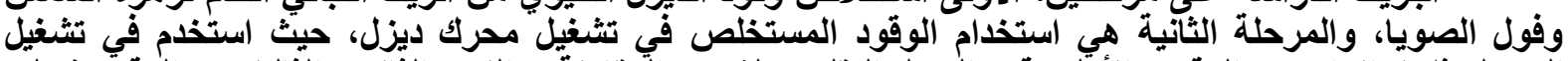

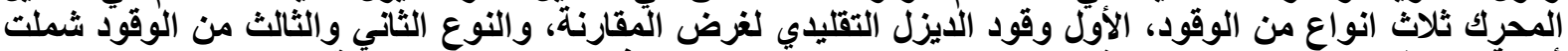

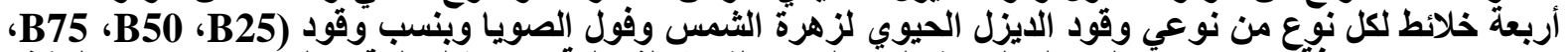

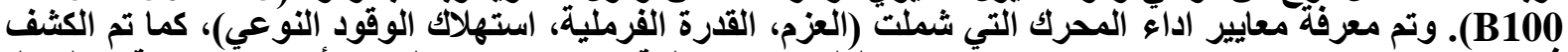

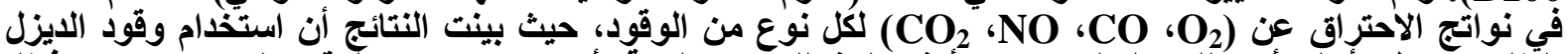

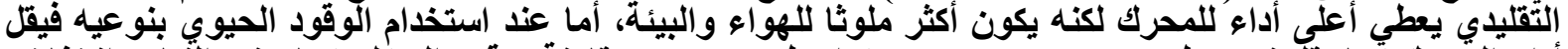

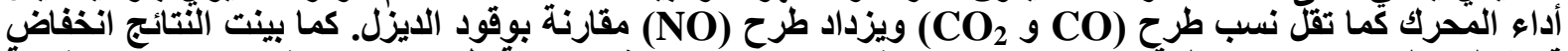

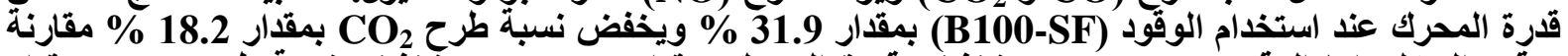

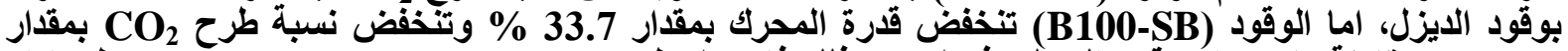

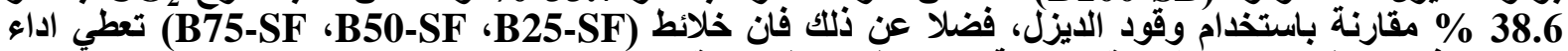
للمحرك افضل وطرحها للملوثات اقل مقارنة بالخلائط المناظرة للوقود (B) (B75-SB ، B50-SB ، B25-SB).

$$
\text { الكلمات المفتاحية: الوقود البديل ، الوقود الحيوي ، محركات احتراق داخلي بلفي }
$$

\title{
Evaluating and Comparing of a Diesel Engine Performance and Its Pollutants Operating on Blends of Sunflower and Soybean Biodiesel Fuel
}

\author{
Arkan M. A. Sedeeq Firas S. Al-khayatt \\ Adil Abdulah \\ Dept. of Agr. Machines \& Equipment/Agr. \& Forestry College/Mosul University
}

\section{Abstract}

This study eventuated in two stages, the first is to extract biodiesel fuel from crude vegetable oil of sunflower and soybean oils, and the second stage is to use these extracted fuels to operate diesel engine. Three types of fuel used, the first fuel is conventional diesel fuels for comparison, the second and third types of fuel consist of four blends (B25, B50, B75, B100) for each type of sunflower and soybean biodiesel fuels. The fuels were used in the engine to find the criteria of performance of the engine which includes(Torque, Brake power, Specific fuel consumption) and discovers in the combustion products on $\left(\mathrm{O}_{2}, \mathrm{CO}, \mathrm{NO}, \mathrm{CO}_{2}\right)$ for each type of fuel. The result of this study shows that the maximum performance of the engine is reached when the conventional diesel fuel is used, but increased the pollutants in atmosphere. The use of biodiesel fuel in the engine decreases the performance of the engine, but it will decrease emitting of $\left(\mathrm{CO}_{2}, \mathrm{CO}\right)$ and increase(NO) comparing with conventional diesel fuel. The results show decrease $31.9 \%$ in engine power when use the fuel (B100-SF) and decrease $18.2 \%$ of $\mathrm{CO}_{2}$ comparing with conventional diesel fuel. The fuel(B100-SB) shows decrease $33.7 \%$ in engine power and decrease $38.6 \%$ of $\mathrm{CO}_{2}$ when comparing with conventional diesel fuel. In addition the blends (B25-SF, B50-SF, B75-SF) give better engine performance and less pollutants emission when comparing with identical blends of fuel (B25-SB, B50-SB, B75-SB). 


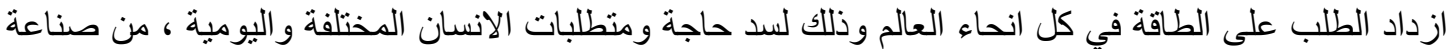

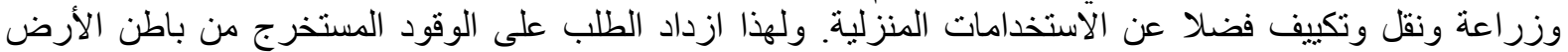

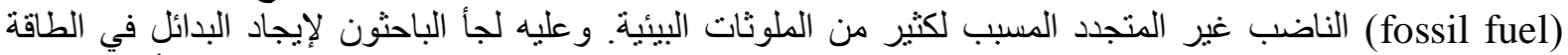

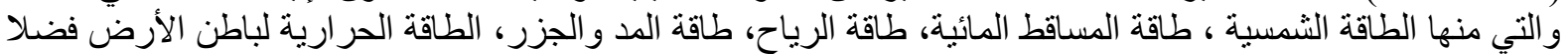

عن طاقة الوقود الحيوي (Bio-fuel).

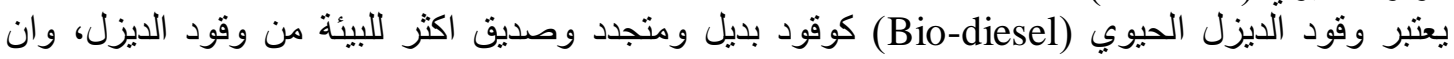

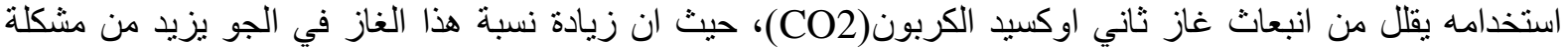

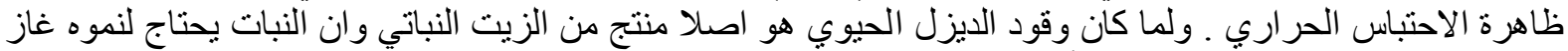

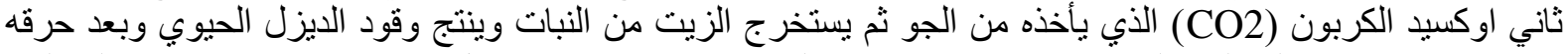

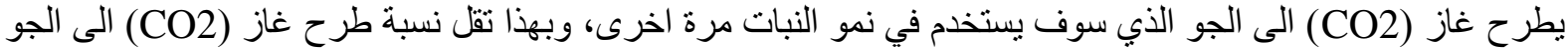

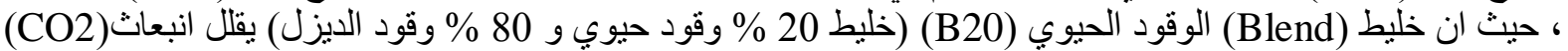

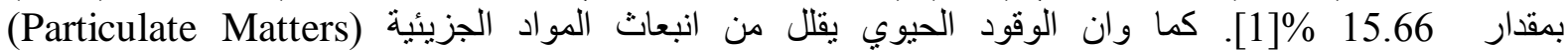
و الهيدروكاربونات (HC) السامين المنبعثين من حرق وقود الديزل واللانان يؤثران على صحة الانسان مسببين السرطان

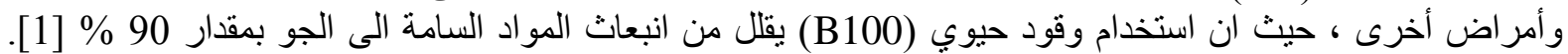

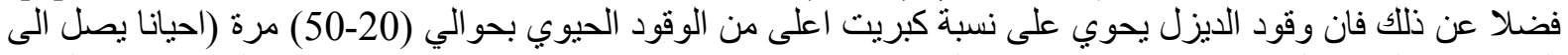

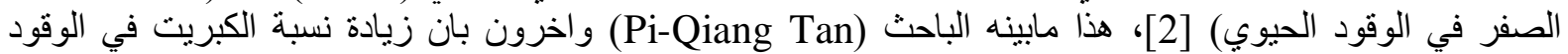

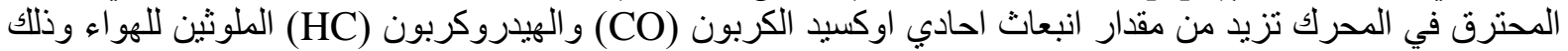

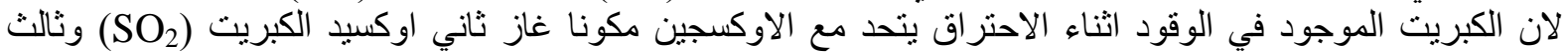

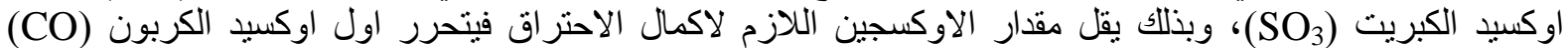

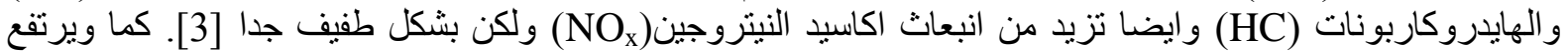

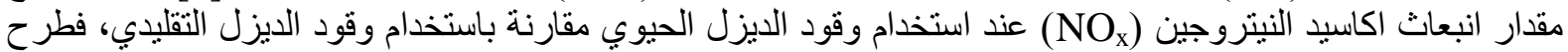

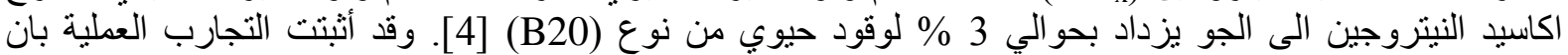

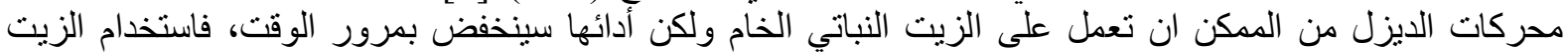

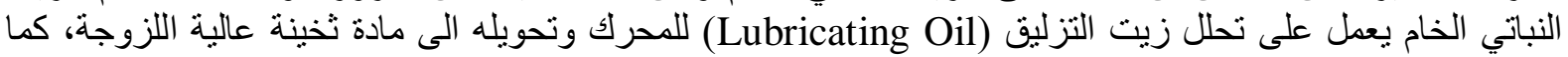

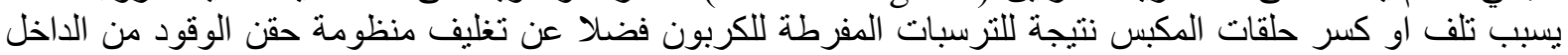

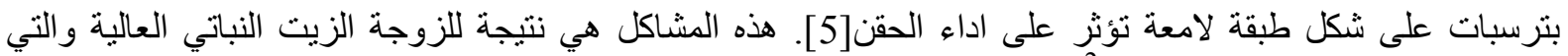

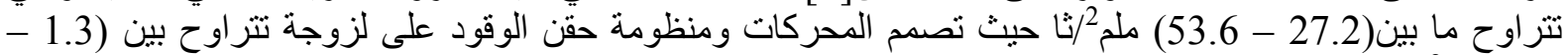

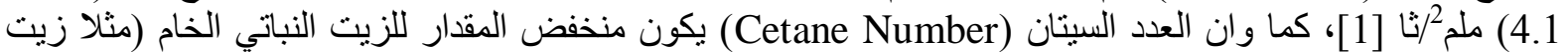

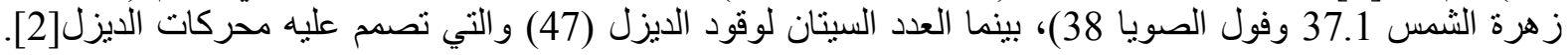

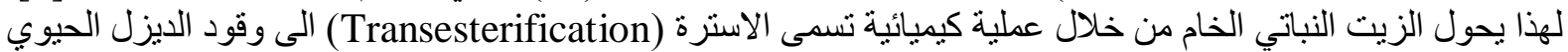

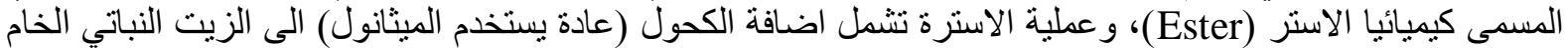

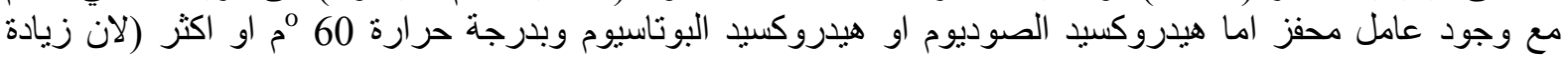

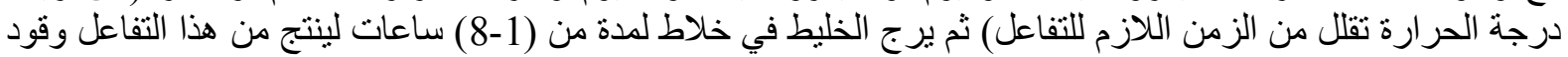

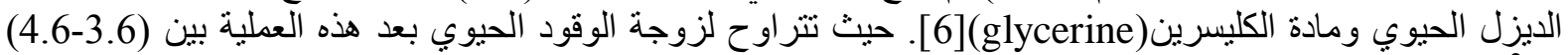

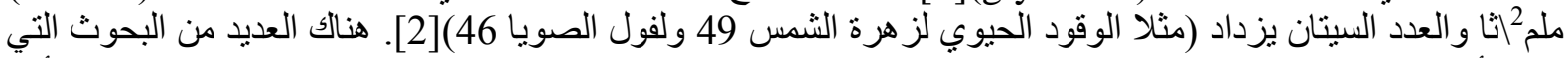

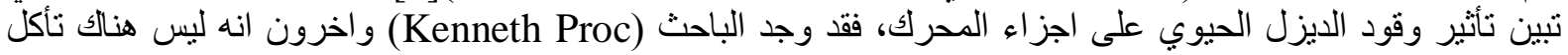

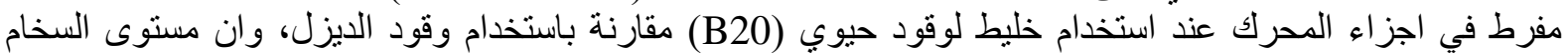

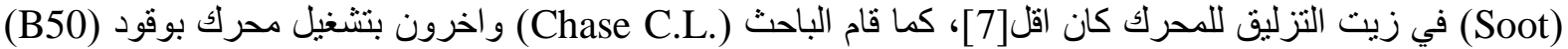

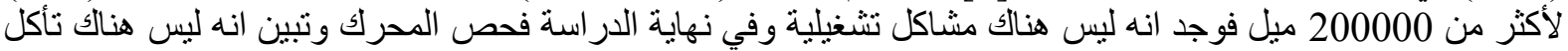

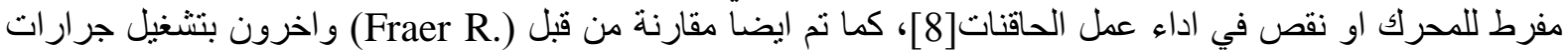

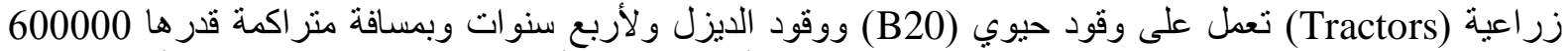

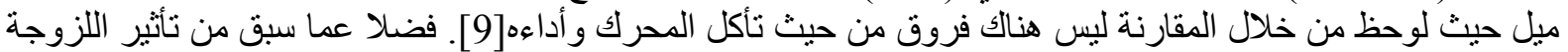

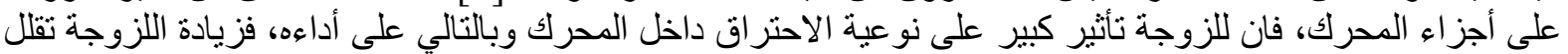

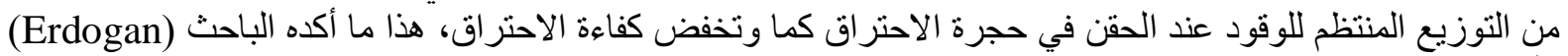

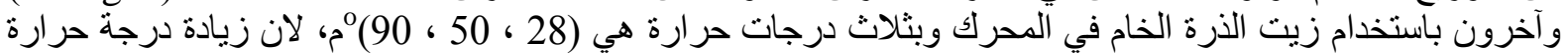
الوقود تخفض اللزوجة حيث كانت لزوجة الزيت الزيت عند درجات الحرارة الثثلاثة وعلى التوالي هي (34.1، 16 ، 10.8 
ملم²ثا، فأظهرت نتائجه ان استخدام الوقود بدرجة حرارة 90 مُ م اعطى اكبر قدرة وعزم مقارنة بالوقود عند درجات

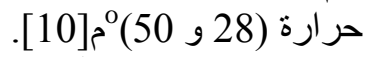

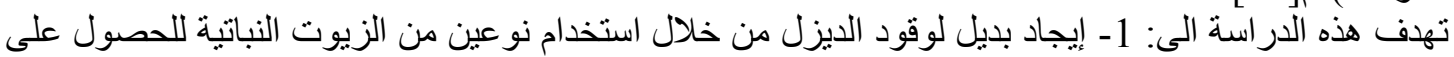

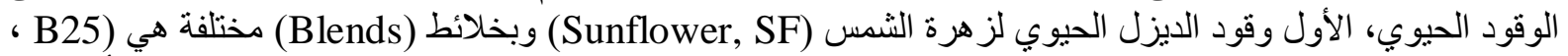

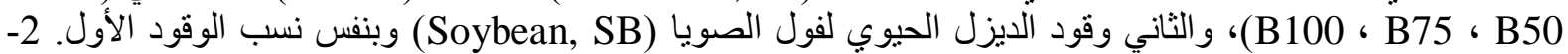

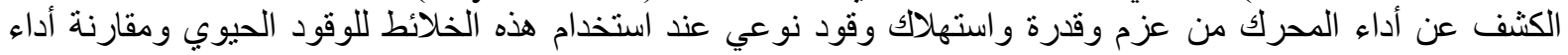

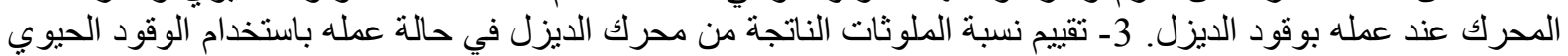
ومقارنة ذللك عند عمله بوقود الديزل.

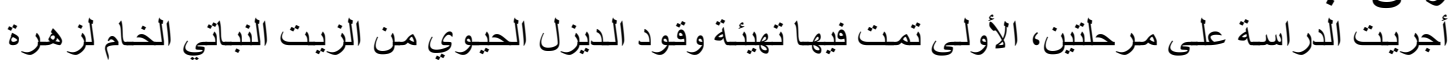

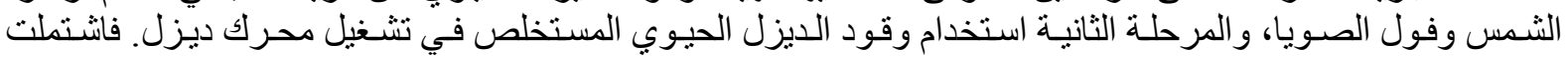

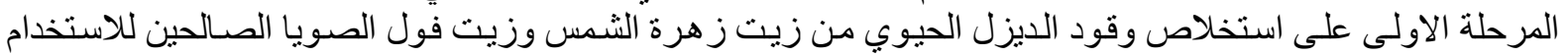

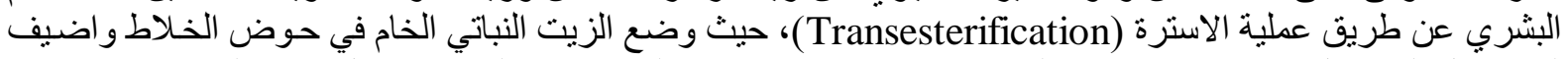

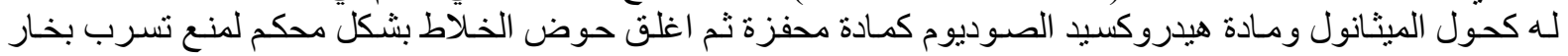

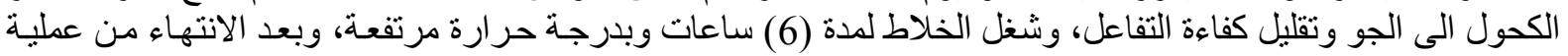

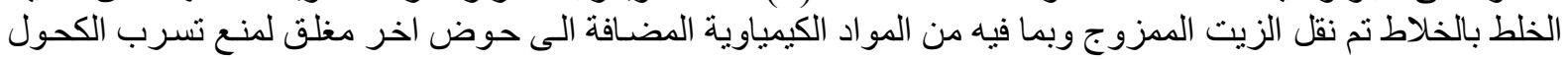

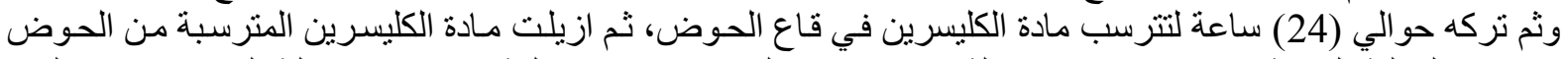

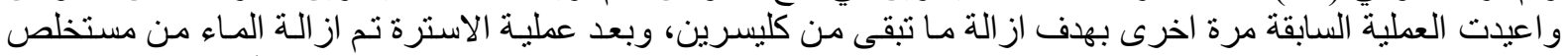

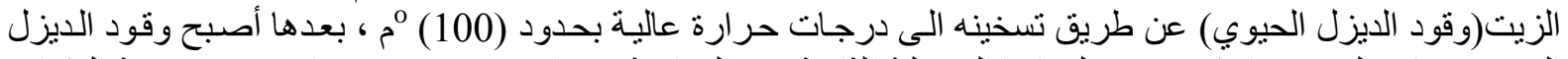

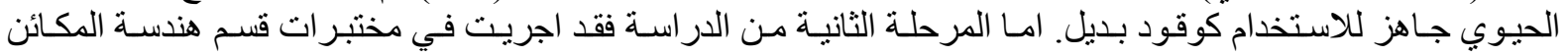

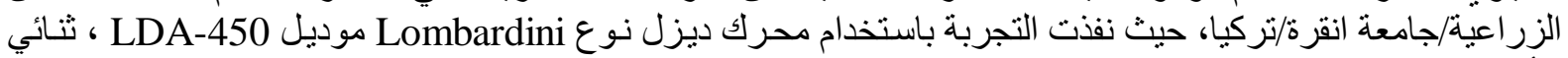

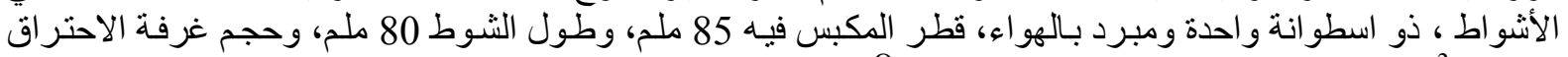

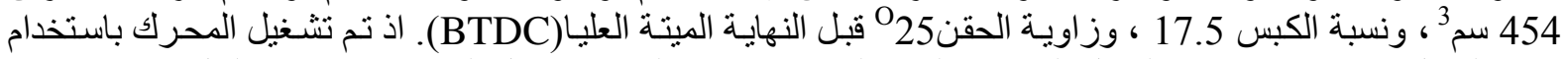

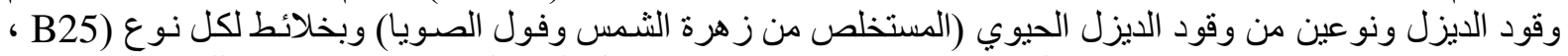

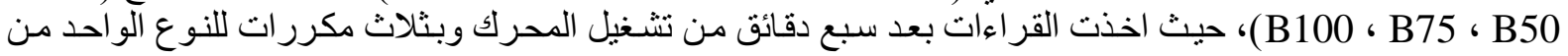

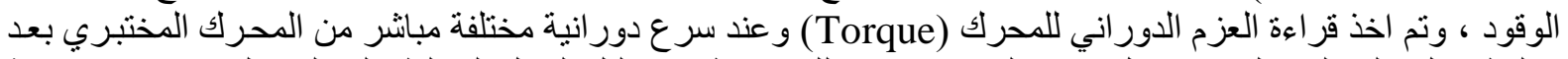

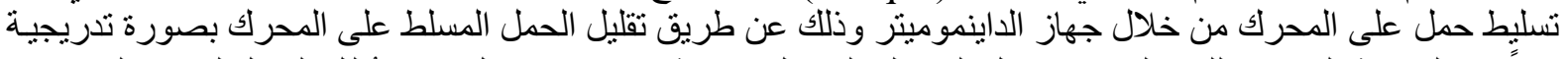

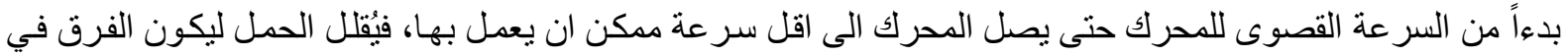

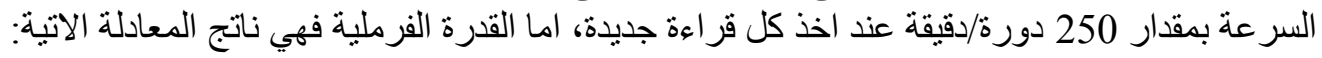

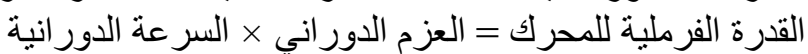

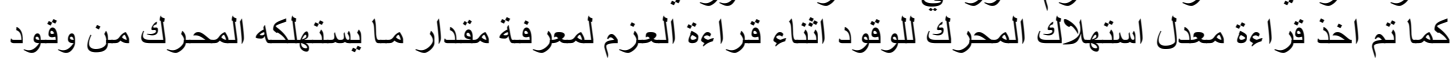

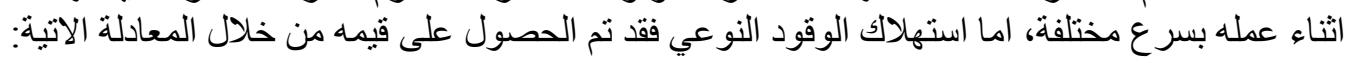

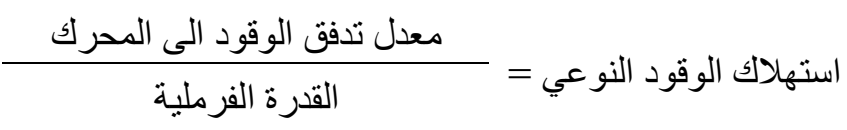

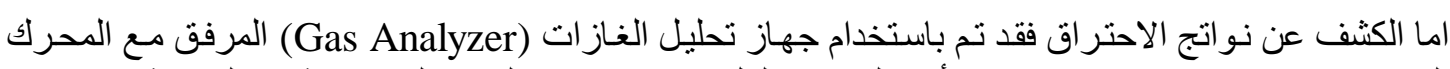
المختبري لقياس (

النتائج والمناقشة

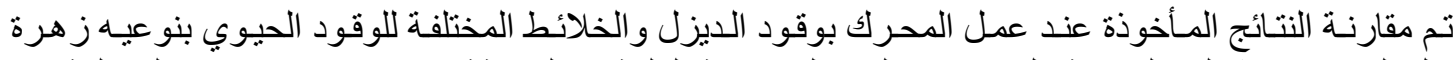

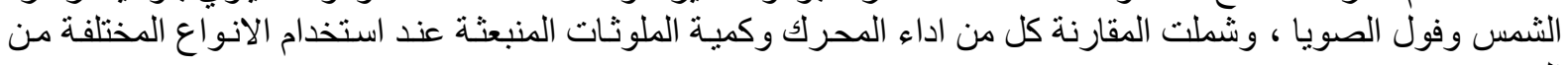

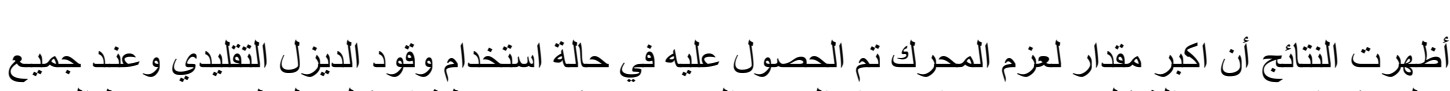
الوقود.

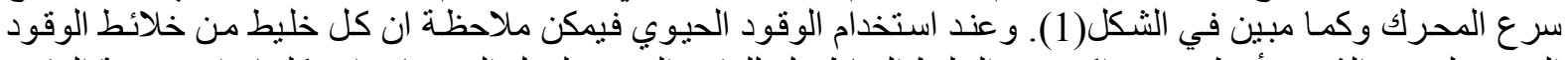

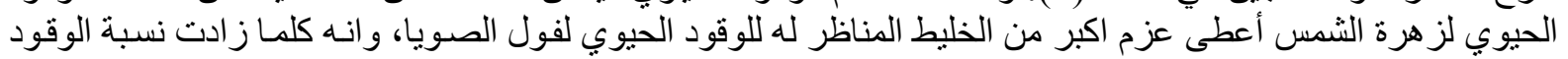




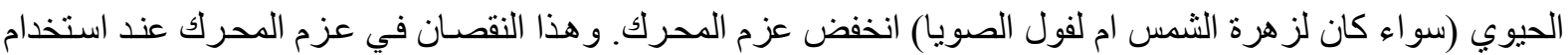

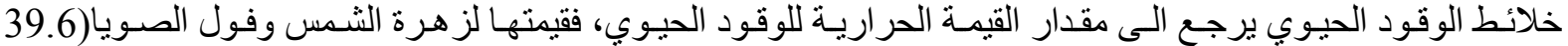

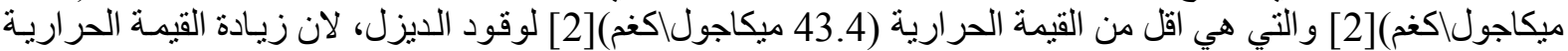
تعمل على زيادة رفع الضغط ودرجة الحرارة داخل حجرة الاحتراق ليتكون قوة اكبر على المكبس لتوليد عزم دوران

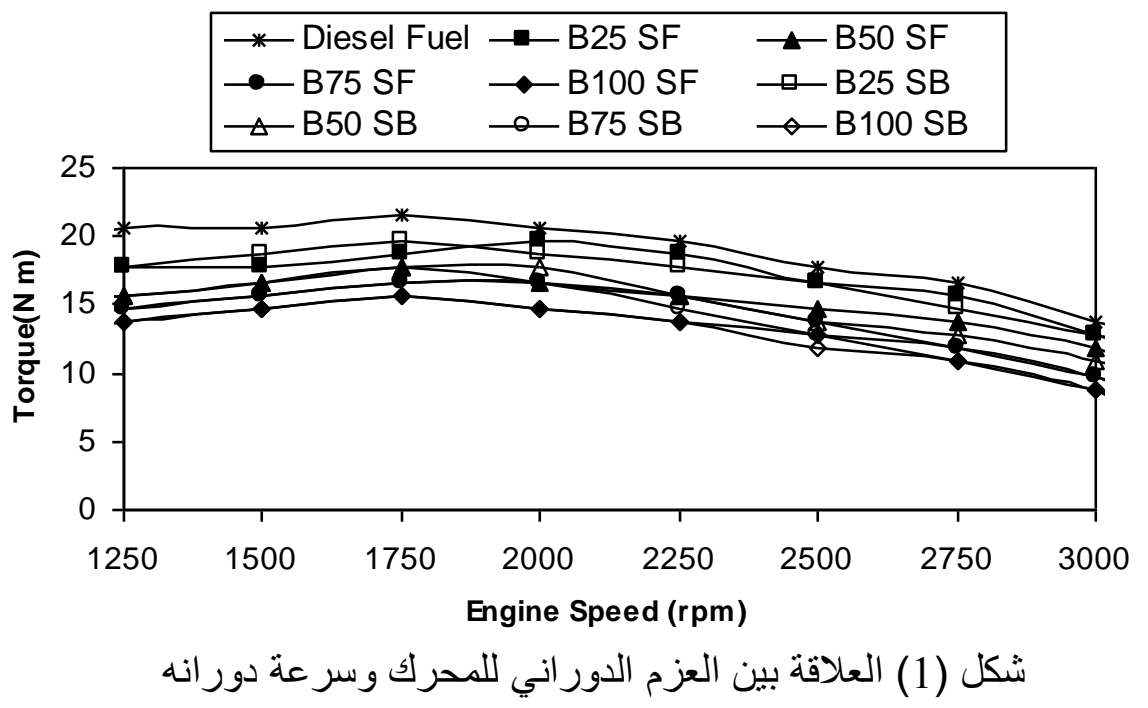

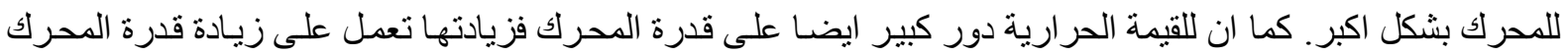

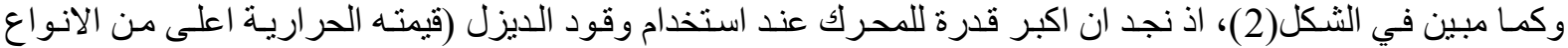

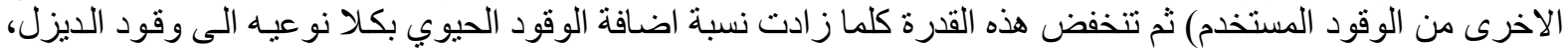

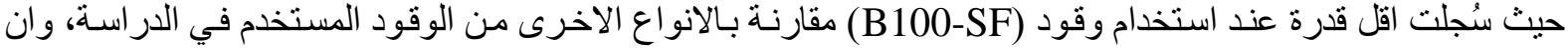

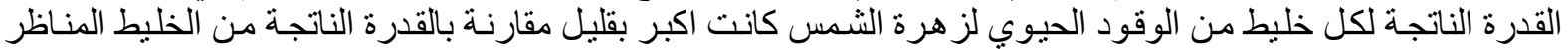

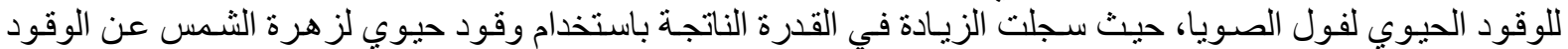

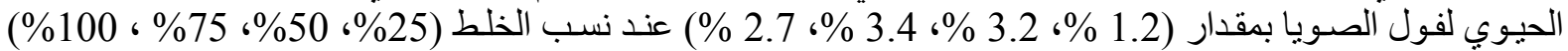

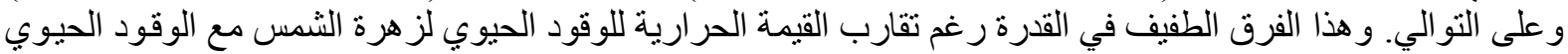

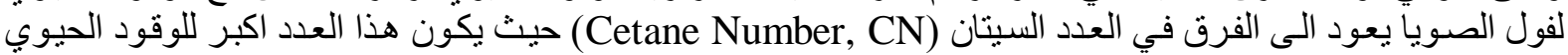

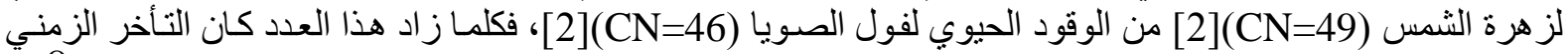

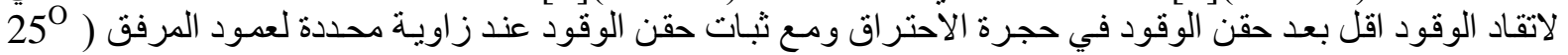

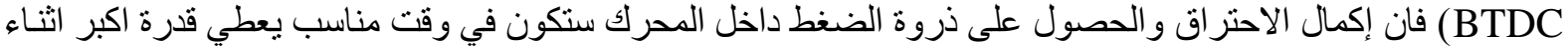
شوط القدرة (Power Stroke) فيكون هذا الفرق الطفيف في القدرة.

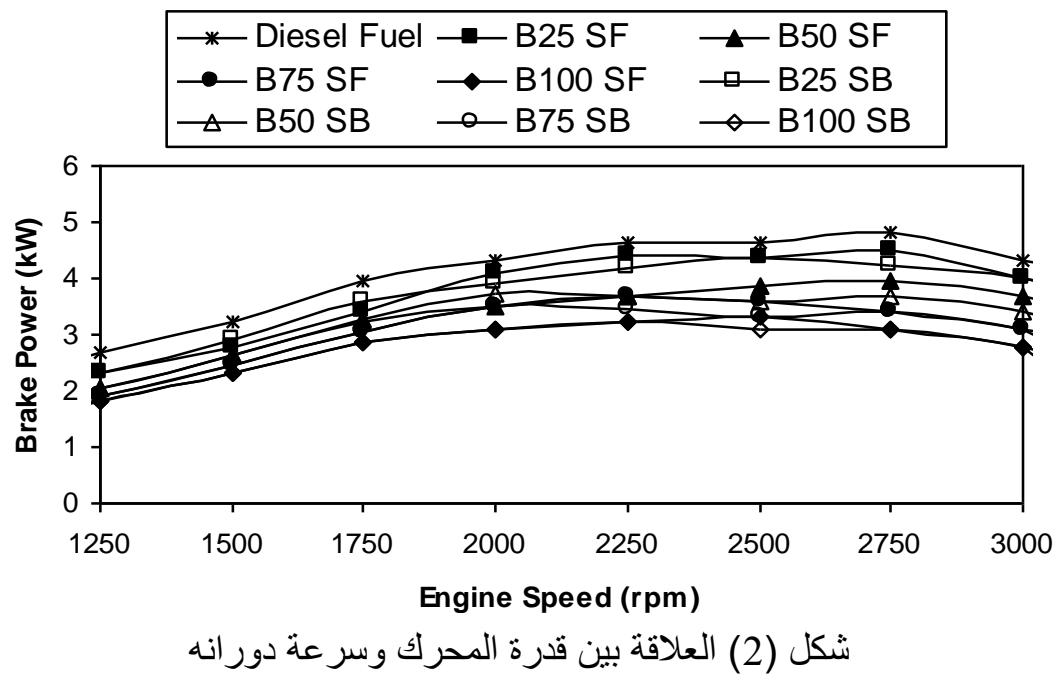


ومن معايير اداء المحرك الأخرى هي استهلاك الوقود النوعي حيث أعطى وقود الديزل اقل استهلاك نوعي

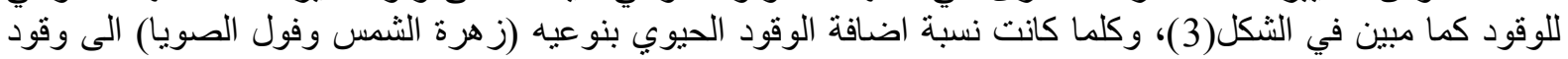

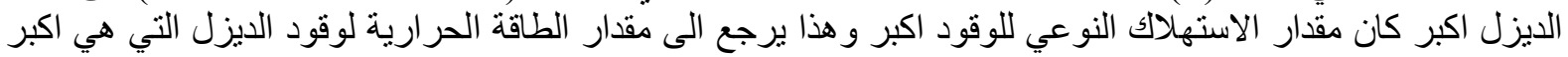

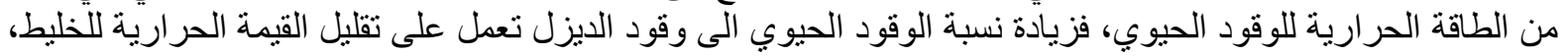

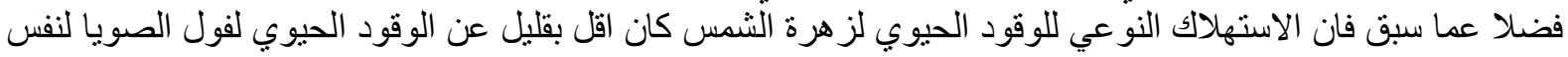

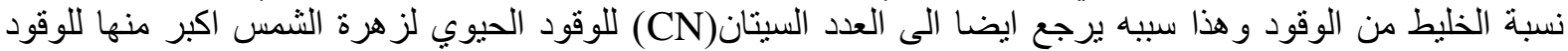

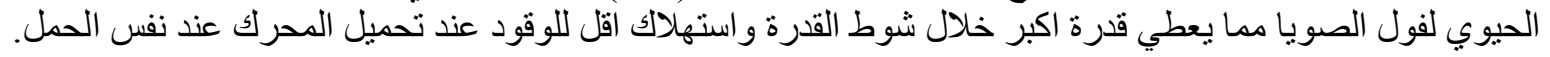

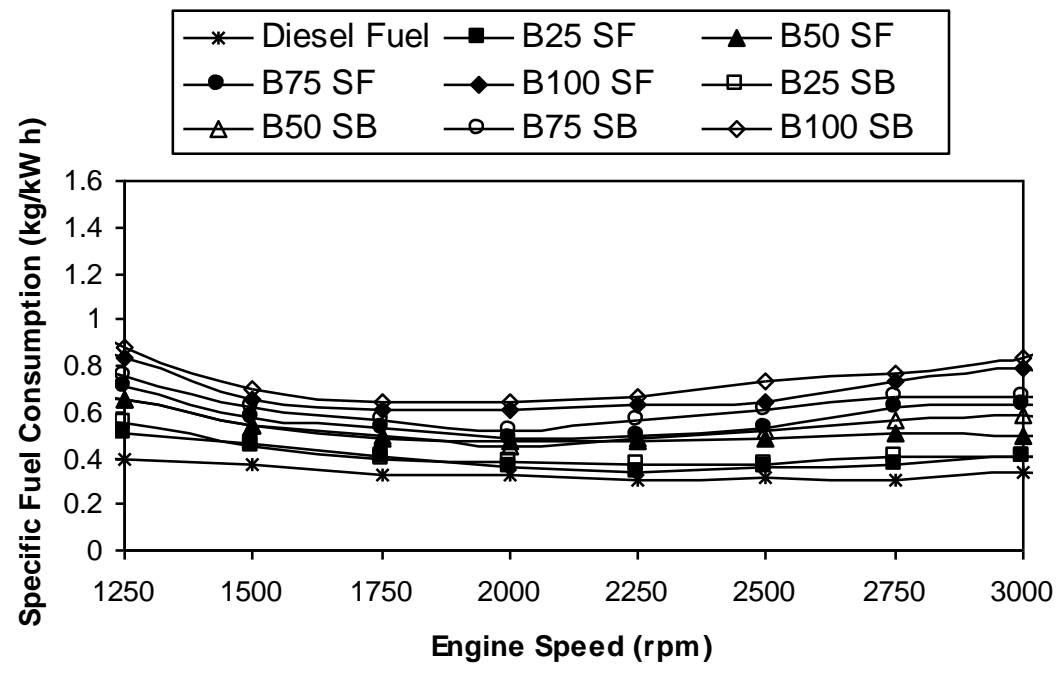

$$
\text { شكل (3) العلاقة بين استهلاك الوقود النوعي المحرك وسر عة دور انه }
$$

وبعد الكثف عن اداء المحرك باستخدام خلائط مختلفة لنو عين من الوقود الحيوي (زهرة الثمس وفول الصول الصويا)

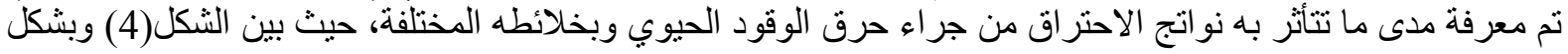

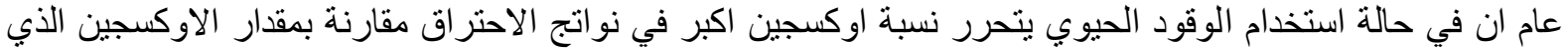

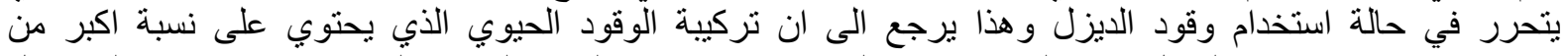

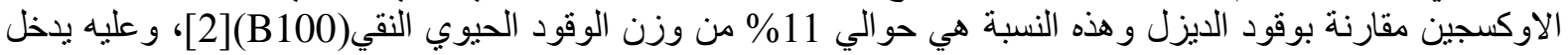

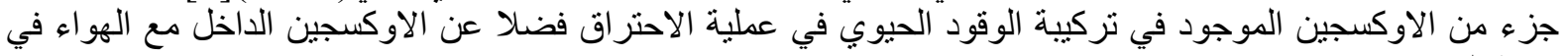
شوط التغذية (Intake Stroke)،

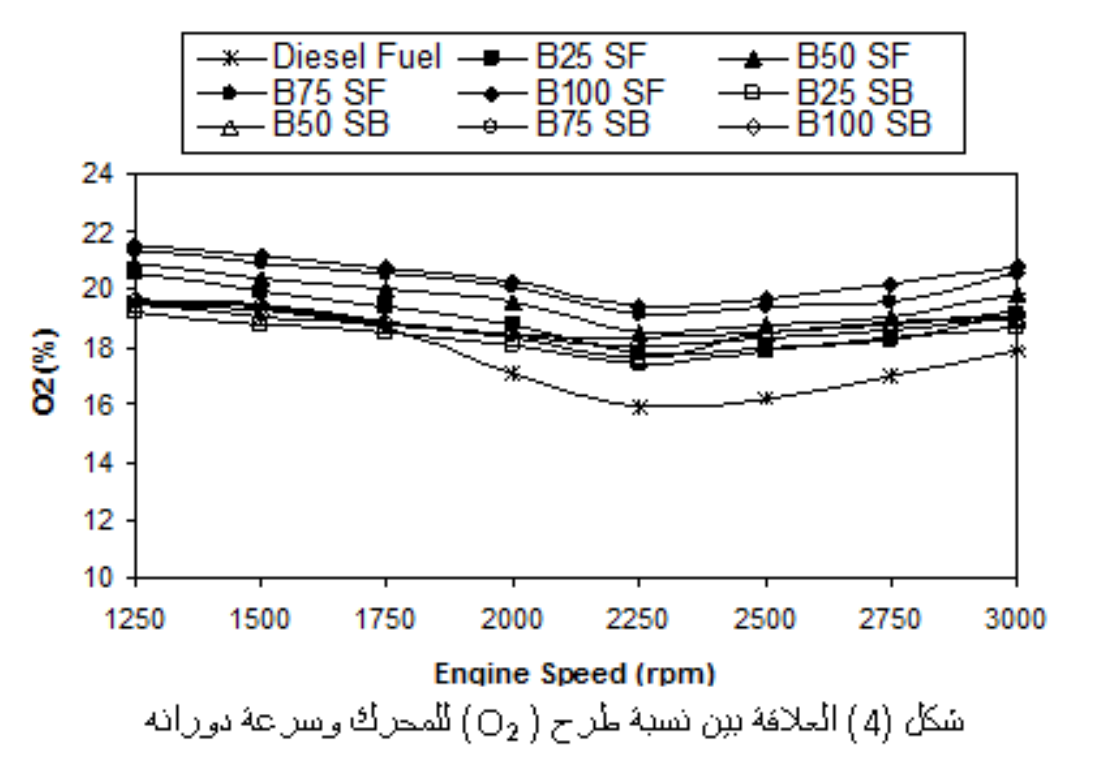




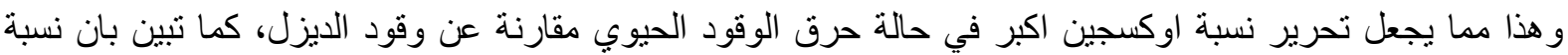

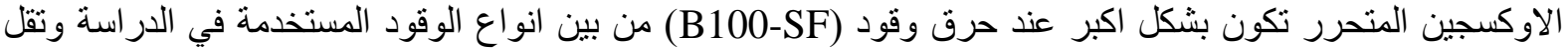

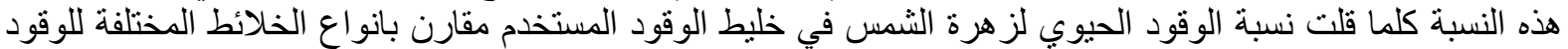

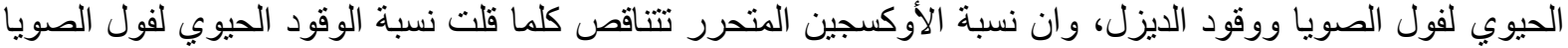

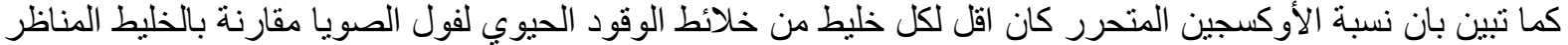

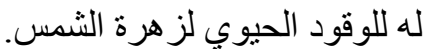

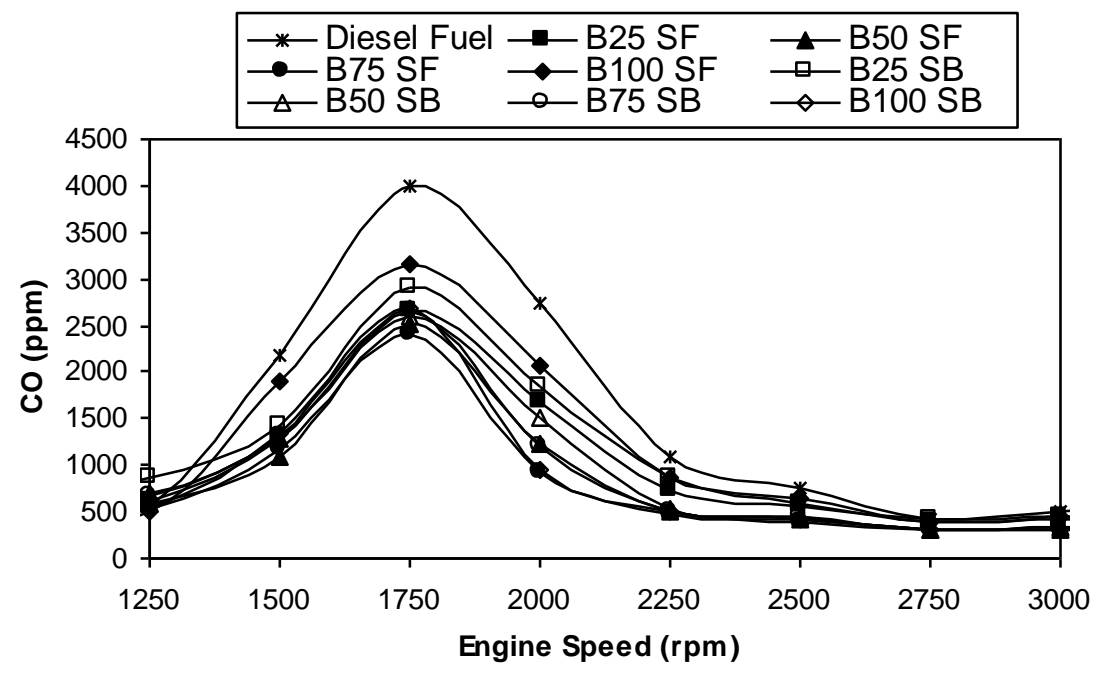

$$
\text { شكل (5) العلاقة بين نسبة طرح (CO) للمحرك وسرعة دور انه }
$$

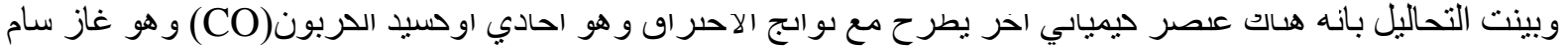

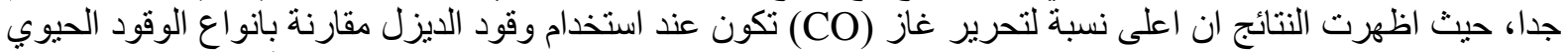

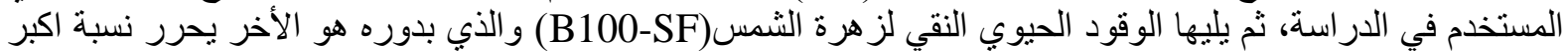

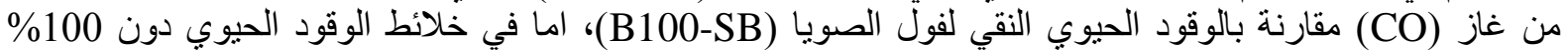

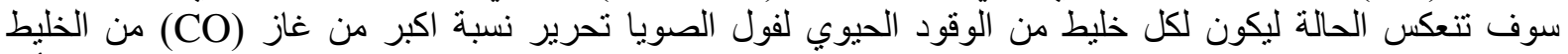

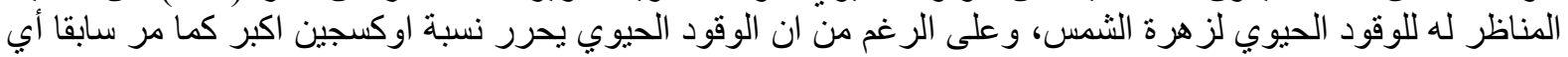

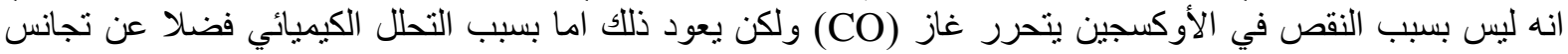

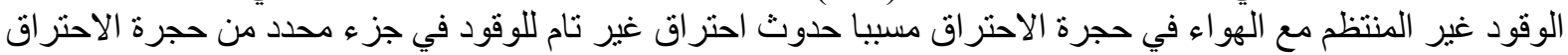

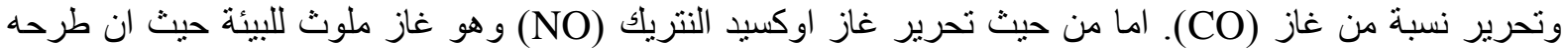

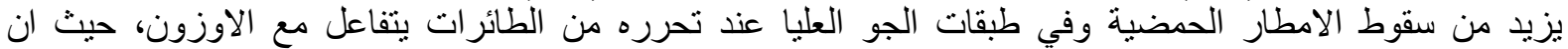
الثكل(6) يوضح ان اقل نسبة تحرر من هذا الغاز في نو اتج الاحتراق كانت عند استخدام وقود الديزل.

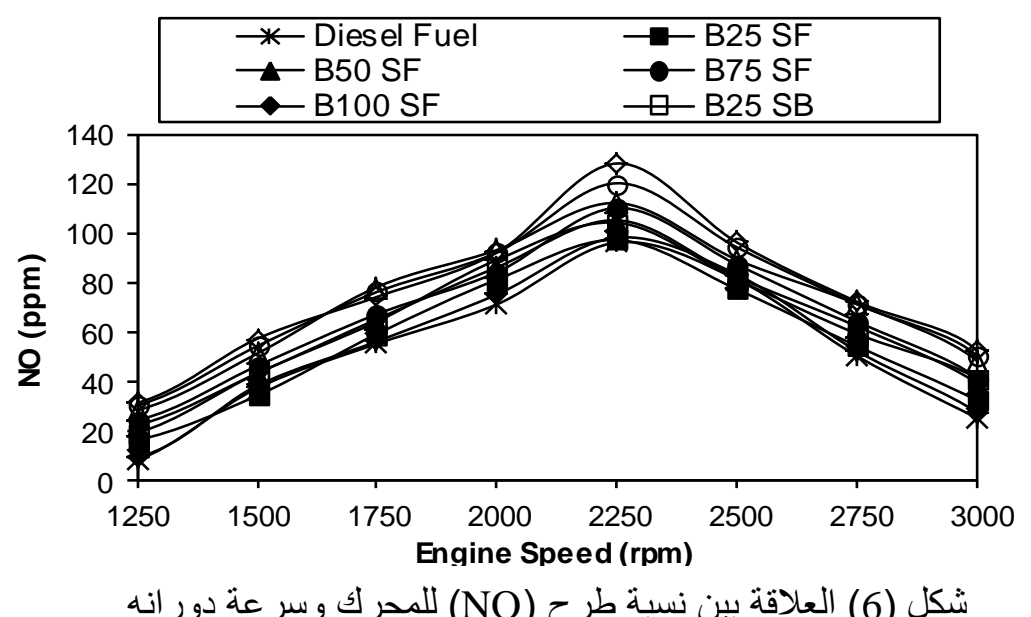


وان حرق الوقود الحيوي بنوعيه يحرر اكبر نسبة من غاز (NO) مقارنة بوقود الديزل، كما وان الوقود الحيوي النقي

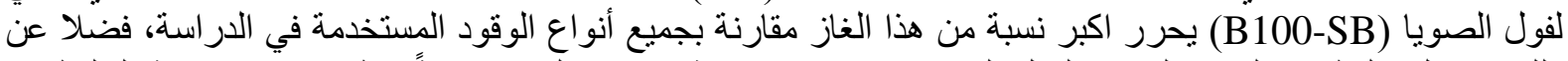

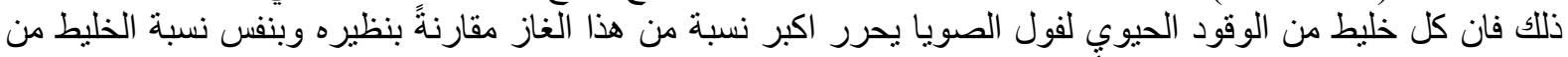

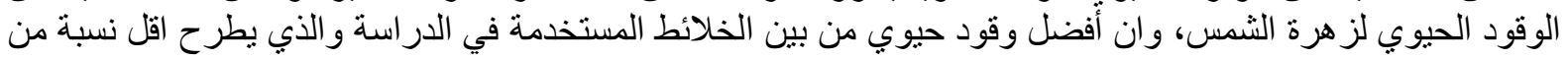

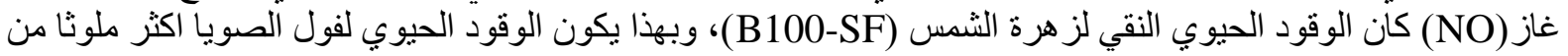

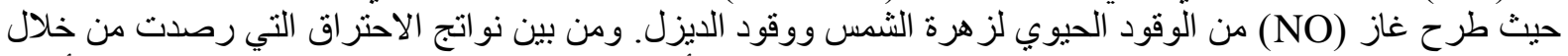

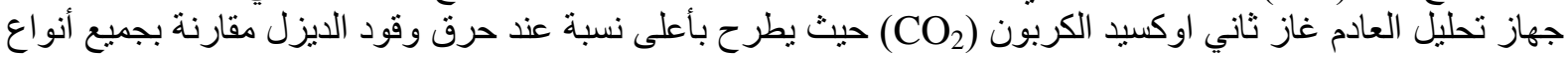

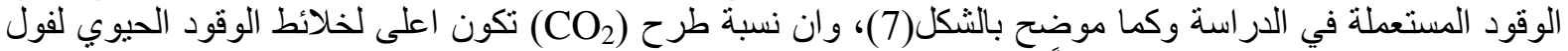

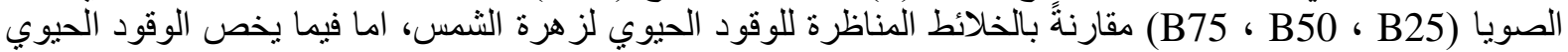

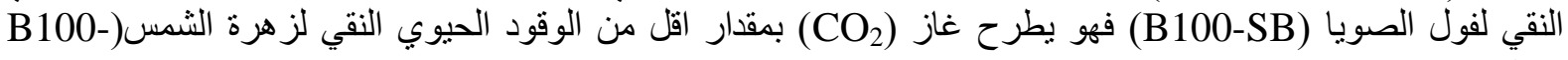

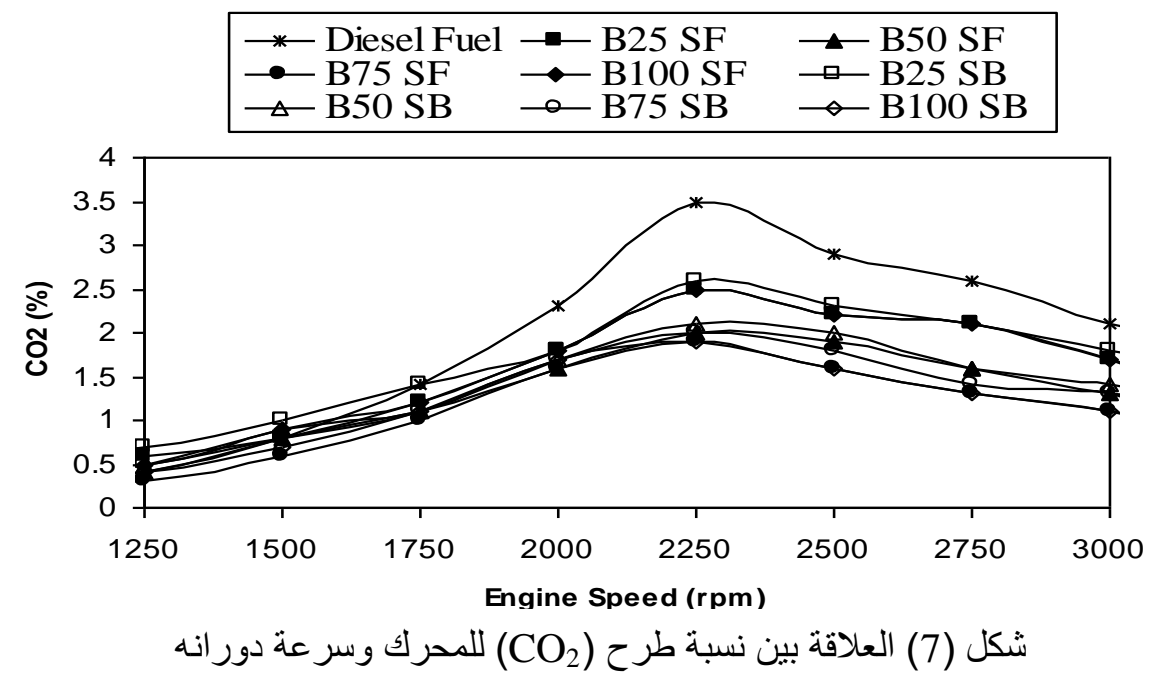

.(SF

و الجدول الأتي يوضح نسب الزيادة والنقصان في معايير الأداء ونواتج الاحتراق للمحرك عند استخدام وقود حيوي لزهرة الثمس وفول الصويا مقارنة بوقود الديزل:

\begin{tabular}{|c|c|c|c|c|c|c|c|}
\hline \multicolumn{4}{|c|}{ نواتج الاحتراق (\%) } & \multicolumn{3}{|c|}{ معايير الأداء المدروسة للمحرك(\%) } & \multirow{2}{*}{ وقود الديزل } \\
\hline $\mathrm{CO}_{2}$ & NO & $\mathrm{CO}$ & $\mathbf{O}_{2}$ & استهلال الوقود & القدرة & العزم & \\
\hline-17.6796 & +8.7356 & -29.8584 & +6.9119 & +19.9683 & -8.4999 & -9.1892 & زهرة الشمس \\
\hline-32.5967 & +22.069 & -41.1152 & +10.5351 & +55.2973 & -18.6705 & -18.9189 & زهرة الشمس \\
\hline-40.884 & +26.8966 & -45.2522 & +13.6009 & +77.0265 & -24.9713 & -24.3243 & زهرة الشمس \\
\hline-18.232 & +3.908 & -17.912 & +15.3289 & +112.6548 & -31.9139 & -30.8108 & زهرة لشمس \\
\hline-12.1547 & +20.9195 & -21.5769 & +3.4002 & +26.8269 & -9.6266 & -9.7352 & فول الصويا \\
\hline-28.7293 & +38.3908 & -34.3851 & +5.2397 & +63.3269 & -21.2231 & -20.4303 & فول الصويا \\
\hline-33.7017 & +42.2989 & -37.1955 & +5.6299 & +94.2742 & -27.4751 & -26.5526 & فول الصويا \\
\hline-38.674 & +45.7471 & -40.6056 & +6.2988 & +136.8756 & -33.7214 & -32.4324 & $\begin{array}{c}\text { فول الصويا } \\
\text { (B100-SB) }\end{array}$ \\
\hline
\end{tabular}

اشارة(+) تعني الزيادة في الصفة المدروسة للوقود الحيوي عن وقود الديزل، واشارة(-) تعني النقصان 
استخدام وقود الديزل التقليدي يعطي اعلى اداء للمحرك من جميع انواع الوقود الحيوي المستخدم في الدراسة لكنه

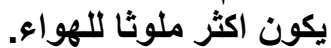
يعطي خليط الوقود الحيوي لزهرة الثئ لئمس (B25-SF) اقل انخفاضا في اداء المحرك من بين خلائط زهرة الثمس

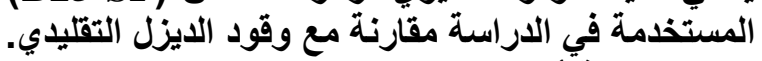

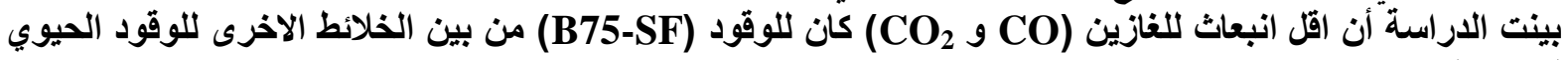
لز لزهرة الثمس. كما واعطى خليط الوقود الحيوي لفول الصويا (B25-SB) اقل انخفاضا في اداء المحرك من بين خلائط فول الصويا وذللك عند المقارنة مع وقود الايزل التول التقليدي. أن اقل انبعاث لغاز (CO و CO2) من حرق خلائط الوقود الحيوي لفول الصويا كان عند استخدام خليط (B100-SB)

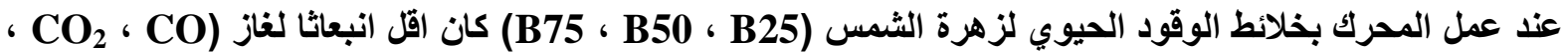

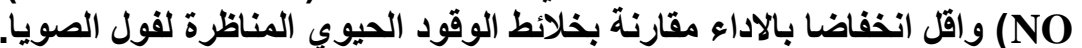

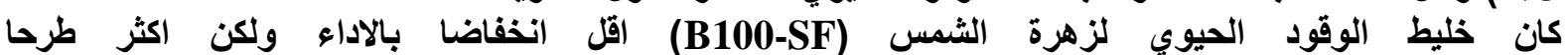

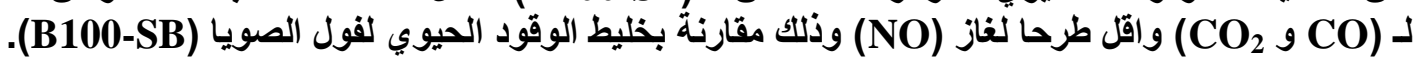

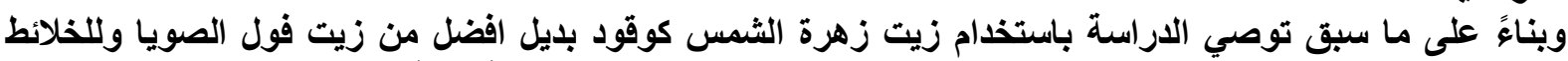

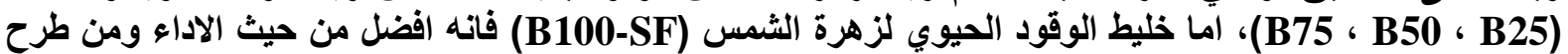

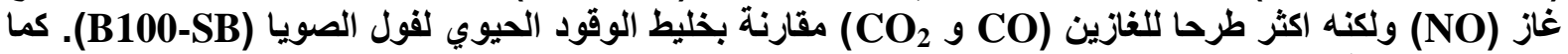

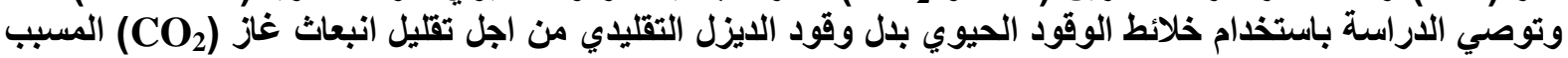

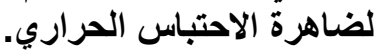

1- K.S. Tyson and R.L. McCormick, "Biodiesel Handling and Use Guide", National Renewable Energy Laboratory - U.S. Department of Energy, Report Number (NREL/TP540-40555), 3rd ed. , 2006.

2- Ayhan Demirbas, "Biodiesel: A Realistic Fuel Alternative for Diesel Engines", SpringerVerlag London Limited, 2008.

3- Pi-Qiang Tan, Zhi-Yuan Hu, Di-Ming Lou, "Regulated and Unregulated Emissions from a Light-Duty Diesel Engine with Different Sulfur Content Fuels", Fuel, Elsevier Ltd., 88(1086-1091)2008. Journal Homepage: www.elsevier.com/locate/fuel.

4- Robert L. McCormick, Christopher J. Tennant, R. Robert Hayes, Stuart Black, John Ireland, Tom McDaniel, Aaron Williams, Mike Frailey, "Regulated Emissions from Biodiesel Tested in Heavy-Duty Engines Meeting 2004 Emission Standards" , National Renewable Energy Laboratory-U.S. Department of Energy, SAE International, Paper No. 2005-01-2200, 2005.

5- Prof. Dr. Gerhard Vogel, "A World Wide Review of the Commercial Production of Biodiesel - A technological, economic and ecological investigation based on case studies", by Mag. Stephan Friedrich, Wien 2004.

6- Herb Willcutt, " Alternative Fuels" , Agr. \& Bio Engineering, Extension Service, Mississippi State University, report (2005).

7- Kenneth Proc, Robb Barnitt, R. Robert Hayes, Matthew Ratcliff, and Robert L. McCormick, "100,000-Mile Evaluation of Transit Buses Operated on Biodiesel Blends (B20)" , National Renewable Energy Laboratory-U.S. Department of Energy, SAE International, Paper No. 2006-01-3253, 2006. 
8- Chase C.L., Peterson C.L., Lowe G.A., Mann P., Smith J.A., Kado N.Y. “A 322,000 Kilometer (200,000 Mile) Over the Road Test with HySEE Biodiesel in a Heavy Duty Truck." SAE Technical Paper No. 2000-01-2647, 2000.

9- Fraer R., Dinh H., Proc K., McCormick R.L., Chandler K., Buchholz B. "Operating Experience and Teardown Analysis for Engines Operated on Biodiesel Blends (B20)." SAE Technical Paper No. 2005-01-3641, 2005.

10- D. Erdogan , A. A. Mohammed, " Effect of Preheated Corn Oil as Fuel on Diesel Engine Performance" , Agricultural Mechanization in Asia, Africa and Latin America, Vol.30 , No.3, 1999.

تم اجراء البحث في كلية ألهنسة = جامعة ألموصل 